\title{
Correlating topography and elastic properties of Elastin-Like Polypeptide scaffolds probed at the nanoscale: Intermodulation Atomic Force Microscopy experiments and Molecular Dynamic simulations
}

\author{
S. Trusso ${ }^{\text {a }}$, S. Firman ${ }^{\mathrm{b}}$, J. Balasubramanian ${ }^{\mathrm{b}}$, M. H. Khatami ${ }^{\mathrm{b}}, \mathrm{H}$. \\ deHaan ${ }^{b}$, N. R. Agarwal ${ }^{b}$ \\ ${ }^{a}$ CNR-Istituto per i Processi Chimico-Fisici, V.le F. Stagno d'Alcontres 37, Faro \\ Superiore, 98158 Messina, Italy \\ ${ }^{b}$ Faculty of Science, University of Ontario Institute of Technology, 2000 Simcoe Street \\ North, Oshawa, ON L1H7K4, Canada.
}

\begin{abstract}
The synthesis and property characterization of soft biomaterials has taken precedence in recent years. Although bulk physical-chemical properties are well known for these bio-materials, nanoscale properties still need to be probed and evaluated to fine tune the bio-compatibility (structural as well as functional) with natural tissues for regenerative medicine, prosthetics and other biological applications. In this study, we focus on a popular soft biomaterial, ELastin-like polypeptide (ELP) which has been prepared under different $\mathrm{pH}$ conditions. We explore the topographical features of the ELP at the nanoscale using Atomic Force Microscopy (AFM). Additionally, we employ a non linear mode of AFM called Intermodulation-AFM (ImAFM) to correlate the elastic properties (Young's modulus) of ELP probed at the nanoscale with the topographical features which gives us a deep insight into the mechanical properties offered by ELP when the structural features are
\end{abstract}


altered by change in the ELP synthesis conditions. The noteworthy point is that we measure theses properties at a spatial resolution of $0.9 \mathrm{~nm}$. Finally, we explain the change in the structural features of ELP with varying $\mathrm{pH}$ through atomistic Molecular Dynamics Simulations. We follow the interaction mechanisms of the amino acid sequences and crosslinkers with proteins as they form the backbone and sidechain of the ELP at different $\mathrm{pH}$.

Keywords: Elastin-like Polypeptide, Intermodulation Atomic Force

Microscopy, Mechanical Properties, Young Modulus, Nanoscale regime, Topography, Soft biomaterials

1. Introduction

The science of synthesizing biomaterials to enhance, support or replace 3 damaged tissue or body functions is at least five decades old. They can either 4 be natural or engineered to gear their function to specific needs. The devel5 opment of biomaterial is a vital area in the fabrication of scaffolds. These 6 engineered substrates are studied predominantly for their surface structural 7 features correlating with cellular responses. The engineered substrates tend lular matrix (ECM). Also, they are found to have angular and repetitive surface structures as compared to ECM that have undulating surfaces [1]. To overcome these differences in properties from native ECM, elastin-based material have been introduced into these engineered substrates since it is one of the most abundant protein found in native ECM [2]. Elastin is a protein that possesses inherent chemical resistance and high durability in entire body owing to the presence of more hydrophobic amino acids [3]. Elastin 
acts as a main component to induce rubber-like elastic nature to the tissues, that support them by sustaining infinite deformation/relaxation cycles without getting ruptured [4]. This property of providing elastic recoil to stiff substrates makes elastin a better choice of material as a component in biomaterial fabrication [5]. Additionally, elastin facilitates accelerated skin wound repair, enhances the proliferation of endothelial cells, vascular smooth muscle cells and skin fibroblast since it has the ability to interact with large number of cell surface receptors [6]. However, the extensive cross-linked structure and hydrophobic nature of elastin hinders the processing and fabrication of elastin into biomaterials [7]. Therefore, recombinant, synthetic and soluble forms of elastin like alpha-elastin, elastin-like polypeptides (ELPs) and tropoelastin are used to fabricate composites scaffolds [8].

Synthetic elastins such as ELPs belong to artificial peptide polymers class. The general composition of ELPs especially Val-Pro-Gly-X-Gly is a repeated unit of pentapeptide, where $\mathrm{X}$ can be any amino acid except proline. The Val-Pro-Gly-X-Gly domain is derived from tropoelastins hydrophobic domain [9]. ELPs are biodegradable and biocompatible. These ELPs exhibit lower critical solution temperature (LCST) phase behavior which results in insoluble coacervate phase formation similar to tropoelastin [10]. The alteration of stimuli such as light, $\mathrm{pH}$, concentration of salts and proteins can tune the LCST of ELPs. The stimuli-triggered self-assembly of ELPs can be achieved by their reversible phase behavior. The ELPs can also self assemble to form hydrogels [11] and nanoparticles [12] that act as potential candidates to use in drug delivery and tissue engineering applications [13, 14]. As an added advantage, the purification of ELPs without chromatography is easier owing 
to its ability to undergo reversible phase separation when it exceeds tunable transition temperature [15].

The formation of hydrogel can be performed with proper peptide sequence and under specific aqueous conditions (e.g., ionic strength, $\mathrm{pH}$ or temperature) via a self-assembly technique [16]. These polypeptides adapt to form secondary structural $\alpha$-helices and $\beta$-sheets. This happens within peptide chains through intramolecular hydrogen bonding that renders specific conformation based self-assembling nature and bioactivity. The polypeptidebased hydrogels have grabbed huge potential in biomedical applications owing to structural similarity to ECM, enzyme biodegradability, cell adhesive properties and remarkable biocompatibility [17]. Moreover, the hydrogels can enhance cells adhesion, proliferation and differentiation and result in good bioactivity. The change in peptide length or replacement of amino acid residues is useful to alter the biological and physical properties of these hydrogels [18].

The common natural polymers (gelatin, collagen, pectin, gellan gum, chitosan and alginate) derived hydrogels also exhibit structural similarity to tissues, biocompatibility and good degradability. However, drawbacks concerning the suboptimal mechanical stability, immunogenic or inflammatory response still persists [19]. The application of hydrogel is mainly based on the mechanical properties of hydrogels. Indeed, the success and fate of the implant is purely based on the hydrogel mechanical properties that are compatible with host tissue and is an important factor for consideration during design and fabrication of tissue regenerative systems. This should avoid the induced host inflammatory response [20]. The cellular responses are directed 
by the extent of matrix stiffness [21]. The existence of mismatch in stiffness between native tissue and hydrogel often results in local stress that concentrates at the interfaces. Also, the stress can be induced due to the lack of proper adhesion of the gel to the adjacent tissue that disturb relative mobility. The immune cell response by generation of pro-inflammatory signals can cause fibrosis and lead to shear stress [22]. Hence, hydrogels that usually exhibit less stiffness than the neighboring tissue could minimize the stress accumulation, and reduce formation of fibrous capsule. The peptide-based hydrogen biomaterials are appealing in areas of regenerative medicine and tissue engineering applications as they can adapt to the stiffness of tissue microenvironments in vivo [19].

The ELPs mechanical and topographical surface analysis plays a vital role in their development stage. In recent years, dynamic AFM technique has been extended to be driven with multiple frequencies as compared to traditional AFM where the cantilever is driven with a single frequency. Here, excitation of probe and measurement of response occurs at two or more frequencies which allow us to obtain more details on tip-surface interaction [23]. One of the multi-frequency dynamic AFM method is Intermodulation AFM (ImAFM) that employs for capturing of several frequency mixing products by two driving frequencies closer to single frequency cantilever resonance instead of higher eigenmodes or higher harmonics excitation of the cantilever [24]. In ImAFM, same thermal noise measurement is applied to calibrate both the cantilever and deflection sensor. ImAFM is capable of measuring the tip-surface force accurately as a function of the cantilever deflection using this calibration. The route to map the materials elastic response and local 
viscous nature arise from the possibility to measure how both the dissipative and conservative components of the interaction depends on the amplitude of oscillatory motion [25].

In this study, we validate the ImAFM technique by measuring the effective elastic properties i.e. Young's modulus of ELP at the nanoscale in spacings of $1 \mathrm{~nm}$ and correlate them with topographical features. The ELP biomaterial were synthesized at different $\mathrm{pH}$ conditions to observe the change in their nanoscopic structural features and their relative elastic behavior. We also explain the underlying mechanisms for different structural properties of ELP at different $\mathrm{pH}$ through Molecular Dynamic simulations.

\section{Experimental section}

\subsection{Elastin-like Polypeptide (ELP) production and purification}

The construction of the plasmid, the expression of ELP in escherichia coli and the following purification steps have been described in detail elsewhere[1]. Shortly, the pET15b plasmid containing the ELP sequence was transformed with a heatshock at $42^{\circ} \mathrm{C}$ into competent BL21-PLyS e.coli (Invitrogen). The ELP contained either a fibronectin cell binding site (RGD) or a scrambled version of that amino sequence (RDG). The transformed bacteria were cultured in terrific broth containing $100 \mu \mathrm{g} / \mathrm{ml}$ ampicillin until an optical density of 0.8 at $600 \mathrm{~nm}$ has been reached, at which point protein expression was induced with $1 \mathrm{mM}$ isopropyl $\beta$-D-1-thiogalactopyranoside. After 5-7 h of expression, the cells were lysed by freeze-thaw cycles with the addition of DNase and $1 \mathrm{mM}$ phenylmethylsulfphonyl fluoride. From there the protein was recovered by iterative inverse temperature cycling. As a final step, the 
protein is dialyzed three times through a $3.5 \mathrm{kDa}$ membrane at $4^{\circ} \mathrm{C}$ and then lyophylized.

\subsection{Intermodulation Atomic Force Microscopy}

The surface morphology of the films produced was imaged by an Atomic Force Microscope (AFM) by NT-MDT working in tapping mode. Images of the sample surface were acquired on a $5 \times 5 \mu \mathrm{m}^{2}$ area using silicon cantilever from Nanosensors (type NCH-50), working at the resonance frequency $\mathrm{f}=362 \mathrm{KHz}$, tip radius $r<10 \mathrm{~nm}$, quality factor of $\mathrm{Q}=490$, and force constant $34.4 \mathrm{~N} / \mathrm{m}$. During the acquisition of the samples surface topography their elastic properties were acquired using the intermodulation technique. In a standard AFM measurement, the tip is driven at its resonance frequency where the tip oscillation amplitude and phase gather information of the topographical features and nature of the investigated material. A linear response of the tip at the driving frequency is assumed. However, the behaviour of the tip motion is highly non linear when the tip interacts with the sample surface.

Measurements were performed with pixel dimensions of 512 x 512 on areas of $5 \times 5 \mu \mathrm{m}^{2}$ giving us a spatial resolution of about $0.9 \mathrm{~nm}$. Within the same intermodulation measurement both the surface topography and the force curves were obtained for each scanned point. The value of the elastic modulus was then obtained by performing the fitting procedure with the modified DMT model to the force curve as analyzed from the inversion of the intermodulation signals. The fitting procedure is performed within the IMP software suite at each point of the scanned surface. 


\subsection{MD simulation details}

We run all-atom molecular dynamics (MD) simulations of elastin-like polypeptide (ELP) system. Our system contains cell-binding (TVYAVTGRGDSPASSAA) and structural domains ((VPGIG)2VPGKG(VPGIG)2)3, QK peptides (KKLTWQELYQL[K(Ac)]Y[K(Ac)]GI), and THPC, TrHP and TrHPO ligands as cross-linkers. Here we use GROMACS 2016.4 package to run our MD simulations, using CHARMM36 force field. We run three simulations to mimic the system at three different $\mathrm{pH}$ values: $\mathrm{pH} 5,7$, and 9 . The only amino acid in the range that could be affected by the change of $\mathrm{pH}$ in this range are HIS and CYS[30]. Thus, based on the amino acids in our ELP, the $\mathrm{pH}$ range that is introduced to our system does not affect the charge of the proteins. However, the change in the $\mathrm{pH}$ affects the properties of crosslinkers in the system. At lower $\mathrm{pH}$ values ( $\mathrm{pH} 5$ ), the only cross-linkers in the system are THPC, while at higher $\mathrm{pH}$ values THPC changes into TrHP and TrHPO ligands (Fig.1)[31, 32, 33, 34]. The content of each system could be found in Table 1. Each simulation box contains 9 cell binding and structural domain peptides, 16 QK peptides and 200 cross-linkers. At pH 5, all of our cross-linkers are THPC molecules, at pH 7 THPC, TrHP and TrHPO ligands co-exist (1:2:1 ratio) as our cross-linkers and in $\mathrm{pH}$ 9, only TrHP and TrHPO ligands (3:2 ratio) are present. $\mathrm{Cl}$ counterions are used to neutralize our system and TIP3P water molecules are used to solvate the simulation box (Table 1).

The same approach is used to set up and run our simulations for all three systems. After energy minimization, under NVT conditions (NVT: a canonical ensemble, where the number of particles $(\mathrm{N})$, the volume of the 
Table 1: System content for each simulation at different $\mathrm{pH}$ values

\begin{tabular}{|c|c|c|c|}
\hline Simulation box content & $\mathrm{pH} 5$ & $\mathrm{pH} 7$ & $\mathrm{pH} 9$ \\
\hline Cell and structural binding & 9 & 9 & 9 \\
QK-peptide & 16 & 16 & 16 \\
THPC & 200 & 50 & - \\
TrHP & - & 100 & 120 \\
TrHPO & - & 50 & 80 \\
$\mathrm{Cl}^{-}$ & 243 & 93 & 43 \\
TIP3P & 45270 & 45661 & 45824 \\
\hline
\end{tabular}

system (V) and the temperature of the system (T) remains constant) we run brief MD simulation at $\mathrm{T}=300 \mathrm{~K}$ using Nose-Hoover thermostat for 2 ns with time steps of $1 \mathrm{fs}$. Protein positions are restrained in this step to prevent any dramatic structural changes before the production run. Next, we run a brief simulation under NPT condition (NPT: an isothermalisobaric ensemble, where the number of particles $(\mathrm{N})$, the pressure of the system $(\mathrm{P})$ and the temperature of the system ( $\mathrm{T}$ ) remains constant) at $\mathrm{T}=300 \mathrm{~K}$, and pressure $=1 \mathrm{~atm}$, using Parrinello-Rahman and isotropic pressure coupling, with $\tau_{p}=5$ ps and compressibility $=4.5 \times 10^{-5} \mathrm{bar}^{-1}$ for $100 \mathrm{ps}$ with time steps of $2 \mathrm{fs}$, keeping the protein positions restrained. After this short simulation, we start the production run under NPT conditions and using the same conditions as the previous step, without position restraints for 400 ns. Visual Molecular Dynamics (VMD) package and customized $\mathrm{C}++$ codes are used to calculate our results. VMD is also used to visualize our structures. 


\section{Results and Discussion}

\subsection{Elastic Properties Modeling}

The elastic properties of the ELP surface were obtained by modelling the contact mechanism of the AFM probe with the samples surface using the Derjaguin-Muller-Toropov (DMT) model [26], a model suitable in cases where there is low adhesion force and small tip radii. The reconstructed force curves $F(z)$, where $z$ is the tip to surface distance, obtained from the non linear analysis of the tip motion $[27,28]$ can be modeled by the following relations:

$$
F(z):= \begin{cases}-F_{\min } \frac{a_{0}^{2}}{\left(z+a_{0}\right)^{2}}-k_{\text {long }} d & z>-h \\ -F_{\min }+\frac{4}{3} E^{*} \sqrt{-R z^{3}}-k_{\text {long }} d & z<-h\end{cases}
$$

where $h$ is the tip equilibrium position above the surface, $F_{\min }$ is the minimum of the force, $a_{0}$ is the intermolecular distance, $R$ is tip radius and $k_{\text {long }}$ is an additional parameter introduced in the DMT model which takes into account long distance interaction force between the tip and the sample surface. As an example, in Fig. 2 we report the results of the fitting procedure with the DMT model to the experimental curve obtained at a given point on the surface of the ELP sample synthesized at pH3. As it can be seen the different regimes e.g. the attractive and repulsive regions of the force-distance curve, e.g. when the tip is not in contact and after the contact are well reproduced. The elastic modulus $E^{*}$ obtained by the fit in this case was of $0.98 \mathrm{GPa}$.

\subsection{Atomic Force Microscopy}

The morphology represented by the ELP at microscopic scales are 'bead and string' like features [29]. When measuring these features with AFM, 
we observe the beads to be bigger than their probable size while the strings are not visualized at all. In Fig.3 are presented the surface topography of the samples grown at pH3, pH6 and pH9 respectively. This is due to the resolution of the AFM which is presented by the tip while it scans the sample. As it can be clearly seen, the surface morphology presents marked differences as a function of the $\mathrm{pH}$ value. The surface of the $\mathrm{pH} 3$ sample is very smooth when observed at short distances, however it shows strong variations of the surface height along distances of the $\mu m$ order. In fact, a height variation of about $2 \mu \mathrm{m}$ is observed along a surface distance of $5 \mu \mathrm{m}$. As the $\mathrm{pH}$ values increases, a different behaviour is observed: at long distances the surface topography seems to become more flat but at the same time height variation at short distances increases. In order to highlight this behaviour in Fig.4 we report the surface profiles collected along a line on the surface of the samples. As it can be clearly seen the bead size of ELP decreases as we move to higher $\mathrm{pH}$ hence the image for $\mathrm{pH} 9$ shows a smoother surface compared to the image for $\mathrm{pH} 3$.

\subsection{Elastic modulus}

In Fig. 5 are shown the map of the elastic modulus obtained by fitting the force curves resulting from the intermodulation measurements at each point of the samples surface. The value of $E^{*}$ is within the 0-1.2 GPa range for all the samples. Nevertheless null or very low $E^{*}$ values usually correspond to surface points where the force curve could not be properly fitted by the DMT model, probably the consequence of a bad tip-surface interaction. Looking at the maps in Fig.5 the details of the surface topography can be clearly observed, pointing out how the surface morphology, and hence the 
$\mathrm{pH}$ value plays a role in determining the elastic properties of the samples. However, a clear picture of the $E^{*}$ dependence on $\mathrm{pH}$ does not emerge from the maps. To this purpose we report in Fig.6 the histogram of distribution of the elastic modulus in the investigated areas for ELP synthesized at different $\mathrm{pH}$. Looking at the $E^{*}$ distribution function for the $\mathrm{pH} 3$ prepared sample, a continuous distribution of values between 0 and $1.2 \mathrm{GPa}$ is observed. Nevertheless three maxima are present: the first peaked at $E^{*}=0$, whose probable origin has been discussed earlier, the second at about 0.21 $\mathrm{GPa}$ and the third at about 0.6-0.7 GPa. The second and third region can be regarded as soft and relatively hard components respectively. It worth noticing that a continuous tail extends till the maximum observed value . The elastic modulus distribution of the samples prepared at $\mathrm{pH} 6$ and $\mathrm{pH} 9$ show a similar behaviour, with the exception of the drastic decrease of the values around $0 \mathrm{GPa}$, pointing out how the different sample morphologies lead to a better tip-surface interaction as long as the surface become more flat. Also for these samples two maxima are present in the $E^{*}$ frequency distribution peaked at 0.3 and $0.7 \mathrm{GPa}$, confirming the observation of a soft and the relatively hard phases. Thus, a well defined two-phase structure, soft and hard, emerges in the elastic properties of the ELP as long as the $\mathrm{pH}$ value is increased above $\mathrm{pH}$. The origin and the molecular structure of these two phases cannot be easily individuated solely on the basis of the AFM and ImAFM measurements. In order to understand the evolution of the ELP structure at a microscopic level as a function of the $\mathrm{pH}$ values, we performed molecular dynamic simulations of the ELP system to understand the underlying mechanisms for change in structural behavior at varying $\mathrm{pH}$. 
3.4. Role of $p H$ on the micro-structural properties of the ELP explained by MD simulations

\subsubsection{General Interaction with Proteins}

Figure 7 indicates the general initial configuration of our system at $\mathrm{pH}$ 7 and final configuration for $\mathrm{pH} 5, \mathrm{pH} 7$, and $\mathrm{pH} 9$. We can see that in all systems, the proteins self-assemble and interact with the cross-linkers. Moreover, in each system, we have cross-linkers floating in the water, which indicates the possibility of these molecules to solubilize in water.

Our results indicate that the number of contacts between proteins and the cross-linkers in the system is $\mathrm{pH}$ dependent (Fig.8A). At pH 5 we have the least amount of interactions between the cross-linkers (THPC) and the protein units, while at $\mathrm{pH} 7$ this value is increased by more than twice between the mixture of the cross-linkers (THPC, TrHP, and TrHPO) and the proteins. At $\mathrm{pH} 9$ the number of interactions is slightly higher than $\mathrm{pH} 7$. At this $\mathrm{pH}$, the mixture of cross-linkers contains TrHP and TrHPO. Based on the results obtained in our simulations, we do not see any major change in the interactions during the last $200 \mathrm{~ns}$ of the simulations. Thus, we have used the last $200 \mathrm{~ns}$ of the trajectory for calculating the rest of our results in this paper unless stated otherwise.

\subsubsection{Detailed Interactions with amino acids}

pH5: At pH 5, we can see that 30\% of THPC cross-linkers interact with the protein (Fig.8B). Similarly, at pH 7, around 30\% of THPC is interacting with proteins, while more than $50 \%$ of TrHPO and $80 \%$ of TrHP is interacting with protein (Fig.8C). At pH 9 TrHPO and TrHP are interacting with proteins at almost the same ratio as the $\mathrm{pH} 7$ system (Fig.8D). Different 
cross-linkers interact with different residues on the proteins. At $\mathrm{pH}$ 5, THPC is mainly interacting with ASP and GLU, which are both negatively charged (Fig.9A). This is compatible with the general view that THPC has a positive charge, and thus interacts with negatively charged residues, based on the electrostatic interactions. Here, we can see that THPC is mainly interacting with side chains of the ASP and GLU. More generally, THPC is mainly interacting with the sidechain of negatively charged or polar residues, while for positively charged or non-polar residues, such as ILE, THPC interacts with the backbone of the residue.

pH7: At pH 7, we can see that THPC is mainly interacting with GLU, ASP (similar to the $\mathrm{pH} 5$ system), which are both negatively charged, GLN, which is polar with partial negative charge and ARG, which is positively charged (Fig. 10). More detailed studies indicate that in the case of ARG, THPC is interacting with the backbone part of the amino acid. In this $\mathrm{pH}$, THPC is mainly interacting with the sidechain of the negatively charged residues and polar residues, similar to the $\mathrm{pH} 5$ system (Fig.10B). The two exceptions are TYR, and ALY (acetylated LYS). First, THPC is mainly interacting with the backbone of TYR at $\mathrm{pH} 7$, unlike $\mathrm{pH}$ 5. Second, THPC is mainly interacting with the side chain of ALY, where the amino acid has partial negative charge. In the case of TrHP, the molecule interacts with a variety of charged, polar and non-polar residues, without any main preference. However, the interactions with charged or polar residues are mainly through sidechains, while for non-polar residues the interactions are mainly through the backbone (Fig.10C). We can see that TrHP has significant interaction with the sidechain of positively charged residues compared to THPC. 
TrHPO has generally much higher interactions with charged residues compared to the other cross-linkers, especially in the case of interacting with LYS (Fig. 10A). Similar to THPC and TrHP, TrHPO interacts with the polar and charged amino acids mainly through the sidechain, and for non-polar residues, the TrHPO mainly interacts with the backbone region (Fig. 10D).

pH9: At pH 9, TrHP and TrHPO have similar interaction with the amino acids in the system (Fig. 11). These patterns are also similar to the patterns of TrHP and TrHPO at pH 7 (Fig. 10). Here, we can see that both crosslinkers have higher interactions with charged and polar residues, and these interactions mainly occur with the side chain of the amino-acids (Fig. 11B and $\mathrm{C}$ ).

\subsubsection{Interaction with the backbone}

We can see in Fig. 12 that in the case of interaction with the backbone, all cross-linkers mainly interact with the CO group of the backbone, which has the highest electronegativity. NH group has much less interaction compared to $\mathrm{CO}$ group, while the $\mathrm{C}_{\alpha}$ has the lowest interaction with cross-linkers in the backbone.

\subsubsection{Interaction with proteins}

Figure 13 indicates the normalized share of interaction between crosslinkers and the protein. We can see that the cross linkers have more normalized interactions with QK peptides in the system. Figure 14 indicates the higher number of interactions between cross-linkers and proteins in higher $\mathrm{pH}$ values. Moreover, these results indicate higher interaction between QK peptides and the cross-linkers in all $\mathrm{pH}$ values. 
Based on our simulations, proteins do aggregate, while the cross-linkers covers the outer area of the protein chunks and produce some domain-like configurations (Figure 15). At lower $\mathrm{pH}$ values, a lower number of crosslinkers cover protein surface. Thus, the domains could diffuse and produce larger domains with similar properties. However, at higher $\mathrm{pH}$ values, the higher surface density of the crosslinkers prevents the protein domains to diffuse and hence the bead size reduces.

\section{Conclusion}

We were able to successfully analyze the elastic properties at the nanoscale of soft biomaterials, ELP in our study, probed by AFM tip using the ImAFM. ImAFM has proven its capabilities to measure the visco-elastic properties of soft materials as a new mode of AFM technique. In particular, we measure the elastic properties of the proteins and not of crosslinkers. The spatial resolution offered by ImAFM to evaluate the elastic properties is about 0.9 $\mathrm{nm}$. Hence, we see that the elastic values at different $\mathrm{pH}$ values remain the same. The domain size dependent on $\mathrm{pH}$ does not change the Youngs modulus value as can be seen in the scale bar (range: 0 - $1.8 \mathrm{GPa}$ ) of the figures with different $\mathrm{pH}$.

Our simulations reach energetically feasible configuration after 200ns of simulations. These simulations indicate direct relation of $\mathrm{pH}$ dependency in ELP systems. Since THPC is positively charged, in all simulations it mainly interacts with negatively charged residues, through electrostatic interactions. TrHPO mainly interacts with polar and charged residues (both positive and negative). This is due to the neutral charge and the $\mathrm{P}=\mathrm{O}$ bond, 
which provides strong H-bond interactions with these residues. TrHP has the highest normalized number of interactions with ELP, which is due to the non-polar region in the center of the particle and $\mathrm{O}-\mathrm{H}$ groups on the chains which provides both polar and non-polar interaction with ELP. In terms of interaction with LYS, TrHPO has the highest interaction, while TrHP has lower interaction and THPC does not interact.

\section{Acknowledgements}

We would like to thank the Natural Sciences and Engineering Research Council of Canada (NSERC) for financial support. The authors are also thankful to Dr. Salvatore Patane for his expertise and providing us with the right stiffness tips from Nanosensors (type NCH-50) to carry out the Intermodulation-AFM experiments. Dr. Agarwal and Dr. Trusso would also like to acknowledge the support of Dr. Daniel Forchheimer and Dr. Erik Tholen for setting-up the Intermodulation system on the NT-MDT AFM hardware. Finally, Dr. Agarwal is grateful to Dr. Alexandra Paul for preparation of the ELP samples at varying $\mathrm{pH}$.

\section{References}

[1] A. Paul, M. Sthrenberg, S. Chen, D. Rhee, W.-K. Lee, T. Odom, S. Heilshorn, A. Enejder, Micro-and nano-patterned elastin-like polypeptide hydrogels for stem cell culture, Soft Matter, 13 (2017) 5665-5675.

[2] Z. Chen, Q. Zhang, H. Li, Q. Wei, X. Zhao, F. Chen, Elastin-like polypeptide modified silk fibroin porous scaffold promotes osteochondral repair, Bioactive materials, 6 (2021) 589-601. 
[3] W. Kim, E.L. Chaikof, Recombinant elastin-mimetic biomaterials: emerging applications in medicine, Advanced drug delivery reviews, 62 (2010) 1468-1478.

[4] F.W. Keeley, C.M. Bellingham, K.A. Woodhouse, Elastin as a selforganizing biomaterial: use of recombinantly expressed human elastin polypeptides as a model for investigations of structure and selfassembly of elastin, Philosophical Transactions of the Royal Society of London. Series B: Biological Sciences, 357 (2002) 185-189.

[5] B. Gurumurthy, P.C. Bierdeman, A.V. Janorkar, Composition of elastin like polypeptidecollagen composite scaffold influences in vitro osteogenic activity of human adipose derived stem cells, Dental Materials, 32 (2016) 1270-1280.

[6] S. Hinderer, S.L. Layland, K. Schenke-Layland, ECM and ECM-like materialsBiomaterials for applications in regenerative medicine and cancer therapy, Advanced drug delivery reviews, 97 (2016) 260-269.

[7] W. Daamen, T. Hafmans, J. Veerkamp, T. Van Kuppevelt, Comparison of five procedures for the purification of insoluble elastin, Biomaterials, 22 (2001) 1997-2005.

[8] D. Miranda-Nieves, E.L. Chaikof, Collagen and elastin biomaterials for the fabrication of engineered living tissues, ACS Biomaterials Science \& Engineering, 3 (2017) 694-711.

[9] V.P. Vaikari, M. Park, L. Keossayan, J.A. MacKay, H. Alachkar, Anti-CD99 scFv-ELP nanoworms for the treatment of acute myeloid 
leukemia, Nanomedicine: Nanotechnology, Biology and Medicine, 29 (2020) 102236.

[10] D. Zhu, H. Wang, P. Trinh, S.C. Heilshorn, F. Yang, Elastin-like proteinhyaluronic acid (ELP-HA) hydrogels with decoupled mechanical and biochemical cues for cartilage regeneration, Biomaterials, 127 (2017) 132-140.

[11] L. Martn, M. Alonso, A. Girotti, F.J. Arias, J.C. Rodrguez-Cabello, Synthesis and characterization of macroporous thermosensitive hydrogels from recombinant elastin-like polymers, Biomacromolecules, 10 (2009) 3015-3022.

[12] J.R. McDaniel, I. Weitzhandler, S. Prevost, K.B. Vargo, M.-S. Appavou, D.A. Hammer, M. Gradzielski, A. Chilkoti, Noncanonical self-assembly of highly asymmetric genetically encoded polypeptide amphiphiles into cylindrical micelles, Nano letters, 14 (2014) 6590-6598.

[13] E.E. Fletcher, D. Yan, A.A. Kosiba, Y. Zhou, H. Shi, Biotechnological applications of elastin-like polypeptides and the inverse transition cycle in the pharmaceutical industry, Protein expression and purification, 153 (2019) 114-120.

[14] S. Roberts, M. Dzuricky, A. Chilkoti, Elastin-like polypeptides as models of intrinsically disordered proteins, FEBS letters, 589 (2015) 2477-2486.

[15] T. Christensen, M. Amiram, S. Dagher, K. TrabbicCarlson, M.F. Shamji, L.A. Setton, A. Chilkoti, Fusion order controls expression level 
and activity of elastinlike polypeptide fusion proteins, Protein Science, 18 (2009) 1377-1387.

[16] H.J. Kang, S. Kumar, A. D’Elia, B. Dash, V. Nanda, H.C. Hsia, M.L. Yarmush, F. Berthiaume, Self-assembled elastin-like polypeptide fusion protein coacervates as competitive inhibitors of advanced glycation endproducts enhance diabetic wound healing, Journal of Controlled Release, 333 (2021) 176-187.

[17] K.J. Hogan, A.G. Mikos, Biodegradable thermoresponsive polymers: Applications in drug delivery and tissue engineering, Polymer, 211 (2020) 123063.

[18] X. Ding, H. Zhao, Y. Li, A.L. Lee, Z. Li, M. Fu, C. Li, Y.Y. Yang, P. Yuan, Synthetic peptide hydrogels as 3D scaffolds for tissue engineering, Advanced drug delivery reviews, (2020).

[19] E. Zeimaran, S. Pourshahrestani, A. Fathi, N.A. bin Abd Razak, N.A. Kadri, A. Sheikhi, F. Baino, Advances in bioactive glass-containing injectable hydrogel biomaterials for tissue regeneration, Acta Biomaterialia, (2021).

[20] S.A. Young, H. Riahinezhad, B.G. Amsden, In situ-forming, mechanically resilient hydrogels for cell delivery, Journal of Materials Chemistry B, 7 (2019) 5742-5761.

[21] R.K. Das, V. Gocheva, R. Hammink, O.F. Zouani, A.E. Rowan, Stressstiffening-mediated stem-cell commitment switch in soft responsive hydrogels, Nature materials, 15 (2016) 318-325. 
[22] R. Klopfleisch, F. Jung, The pathology of the foreign body reaction against biomaterials, Journal of biomedical materials research Part A, 105 (2017) 927-940.

[23] R. Garcia, E.T. Herruzo, The emergence of multifrequency force microscopy, Nature nanotechnology, 7 (2012) 217.

[24] D. Platz, E.A. Tholn, D. Pesen, D.B. Haviland, Intermodulation atomic force microscopy, Applied Physics Letters, 92 (2008) 153106.

[25] H. Huang, I. Dobryden, P.-A. Thorn, L. Ejenstam, J. Pan, M. Fielden, D. Haviland, P.M. Claesson, Local surface mechanical properties of PDMS-silica nanocomposite probed with Intermodulation AFM, Composites Science and Technology, 150 (2017) 111-119.

[26] B.V. Deriaguin, V.M. Muller, Y.P. Toporov, Effect of contact deformation on the adhesion of particles, J. Coll. Interf. Sci. 53, 314 (1975).

[27] D. Forchheimer, D. Platz, E. Tholén, B.B. Haviland, Model-based extraction of material properties in multifrequency atomic force microscopy, Phys. Rev. B, 85, 195449 (2012).

[28] S.S. Borysov, D. Platz, A.S de Wijn, D. Forchheimer, E. Tholén, A.V. Balatsky, B.B. Haviland, Reconstruction of tip-surface interactions with multimodal intermodulation atomic force microscopy, Phys. Rev, B 88, 115405 (2013).

[29] H. Wang, A. Paul, D. Nguyen, A. Enejder, S.C. Heilshorn, Tunable Control of Hydrogel Microstructure by Kinetic Competition between Self- 
Assembly and Crosslinking of Elastin-like Proteins, ACS Appl. Mater. Interfaces. 10, 21808 (2018).

[30] D. L. Nelson and M. M. Cox, Lehninger Principles of Biochemistry, New York: Worth Publishers, 2000.

[31] D. A. Handley, in Colloidal Gold: Principles, Methods and Applications, ed. M. A. Hayat, Academic, San Diego, CA, 1989.

[32] Frank, A. W., \& Drake, G. L. (1973). The Iodometric Determination of P(III) in Flame Retardants for Cotton: Part I: Development of the Method. Textile Research Journal, 43633638.

[33] M. Grayson (1963). Phosphonium Compounds. III. Mechanism of Hydroxide Cleavage of Tetrakis(hydroxymethyl)phosphonium Chloride. Journal of the American Chemical Society, 857983.

[34] S. X. Shao, L. Jiang, Li, Y. Li, Q. K. Shi (2012) Effect of pH on the Phosphorous Components in Tetra-Hydroxymethyl Phosphonium Chloride Solution. Advanced Materials Research, 237241. 


\section{List of Figures}

1 The structures of A) tetra-hydroxymethyl phosphonium chloride (THPC), B) tri-hydroxymethyl phosphine (TrHP), C) trihydroxymethyl phosphine oxide (TrHPO). . . . . . . . . . 26

2 Force-deflection curve acquired on the surface of the pH3 ELP sample. The red curve is the result of the fitting procedure using Eq.1. . . . . . . . . . . . . . . . . . . . . . . 27

$3 \quad$ AFM images (scan area $5 \mu \mathrm{mx} 5 \mu \mathrm{m}$ ) of the surfaces of the ELP sample prepared at a) pH3, b) pH6 and c) pH9. . . . . . . . 28

4 Line profile along the surface of the ELP samples produced at different $\mathrm{pH}$ values. . . . . . . . . . . . . . . . . 29

5 Maps of the elastic modulus $\mathrm{E}^{*}$ obtained fitting the force curves using the DMT model. Maps in panel a), b) and c) correspond to the scanned areas reported in Fig.3. . . . . . . . 30

6 Histogram of distribution function of elastic modulus for ELP synthesized for different $\mathrm{pH} 3,6$ and 9 . . . . . . . . . . 31

7 A) Top and B) side view of the initial configuration of the system for $\mathrm{pH}$ 7. The protein content and position are the same for all three $\mathrm{pH}$ value systems. The cell binding and structural binding sites are covalently connected. The initial configuration of proteins is set to $\alpha$-helical configuration. Cell binding peptides are in orange, structural binding peptides are in blue and QK peptides are in red. LYS amino acids are in green. THPC is in purple, TrHP is in orange and TrHPO is in tan. . . . . . . . . . . . . . . . . . 32

8 Interactions between the cross-linkers and proteins. A) Number of total contacts at different $\mathrm{pH}$ values, $\mathrm{B}$ ) percent of THPC crosslinkers interacting with proteins at $\mathrm{pH} 5, \mathrm{C}$ ) percent of different crosslinkers interacting with proteins at $\mathrm{pH}$ 7 and D) percent of different crosslinkers interactingwith pro-

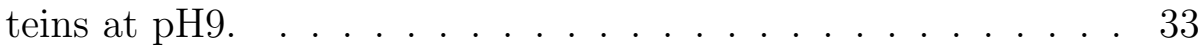


9 Cross-linkers at $\mathrm{pH} 5$ interacting with different amino acids of the protein mixture. A) Number of contacts between THPC cross-linkers and different amino acids residues in the system and $\mathrm{B}$ ) ratio of THPC interacting with the side chain vs backbone of each amino acid. The values in (A) are normalized by the number of amino acids in the system and the number of cross-linkers in the system. . . . . . . . . . . . . . . 34

10 Cross-linkers at $\mathrm{pH} 7$ interacting with different amino acids of the protein mixture. A) Number of contacts between THPC, TrHP and TrHPO cross-linkers and different amino acids residues in the system, B) ratio of THPC interacting with the side chain vs backbone of each amino acid, C) ratio of TrHP interacting with the side chain vs backbone of each amino acid and D) ratio of TrHPO interacting with the side chain vs backbone of each amino acid. The values in (A) are normalized by the number of amino acids in the system and the number of crosslinkers in the system. . . . . . . . . . . . . . . 35

11 Cross-linkers at pH 9 interacting with different amino acids of the protein mixture. A) Number of contacts between TrHP and TrHPO cross-linkers and different amino acids residues in the system, B) ratio of TrHP interacting with the side chain vs backbone of each amino acid and $\mathrm{C}$ ) ratio of TrHPO interacting with the side chain vs backbone of each amino acid. The values in (A) are normalized by the number of each amino acids in the system and the number of cross-linkers in the system. 36

12 Number of contacts between different cross-linkers with different parts of protein backbone in different $\mathrm{pH}$ values. A) $\mathrm{pH}$, B) pH7 and C) pH9. Number of contacts are normalized by total number of the cross-linker. . . . . . . . . . . . . . 37

13 Number of contacts between different cross-linkers and different proteins in the system at different $\mathrm{pH}$ values. A) $\mathrm{pH}$, B) pH7 and C) pH9. Each cross-linker interaction is normalized by total number of the cross-linker. For each peptide, the number of interactions is normalized by number of the same type peptides, as well as the number of amino acids on each peptide. . . . . . . . . . . . . . . 38 
14 Number of contacts between all cross-linkers and different proteins in the system at different $\mathrm{pH}$ values. The values are normalized by total number of the cross-linkers and total the number of amino acids on each peptide. . . . . . . . . . . .

15 Schematics on the role of cross-linkers and proteins in producing the ELP configurations in different $\mathrm{pH}$ values. At low $\mathrm{pH}$ values (A), lower number of cross-linkers have covered the protein, while at higher $\mathrm{pH}$ values, (B) a greater number of cross-linkers have covered the protein. The aggregated proteins are shown as purple circles. The cross-linkers are shown as cross sign in different colors.. . . . . . . . . . . . . . . . . . 40 


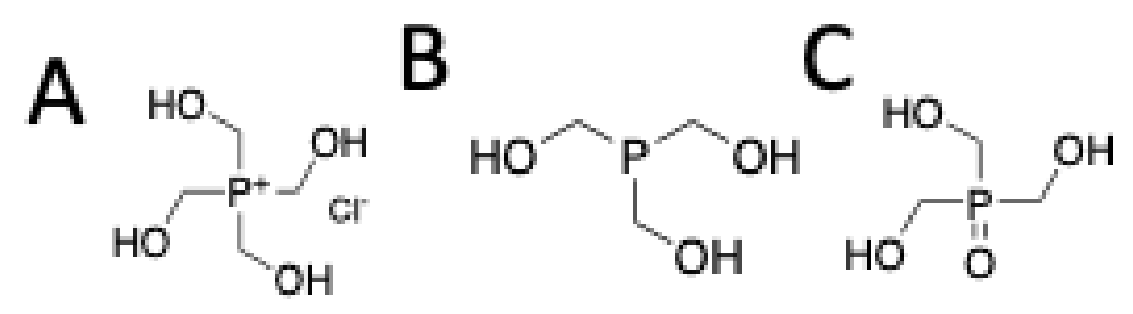

Figure 1: The structures of A) tetra-hydroxymethyl phosphonium chloride (THPC), B) tri-hydroxymethyl phosphine (TrHP), C) tri-hydroxymethyl phosphine oxide (TrHPO). 


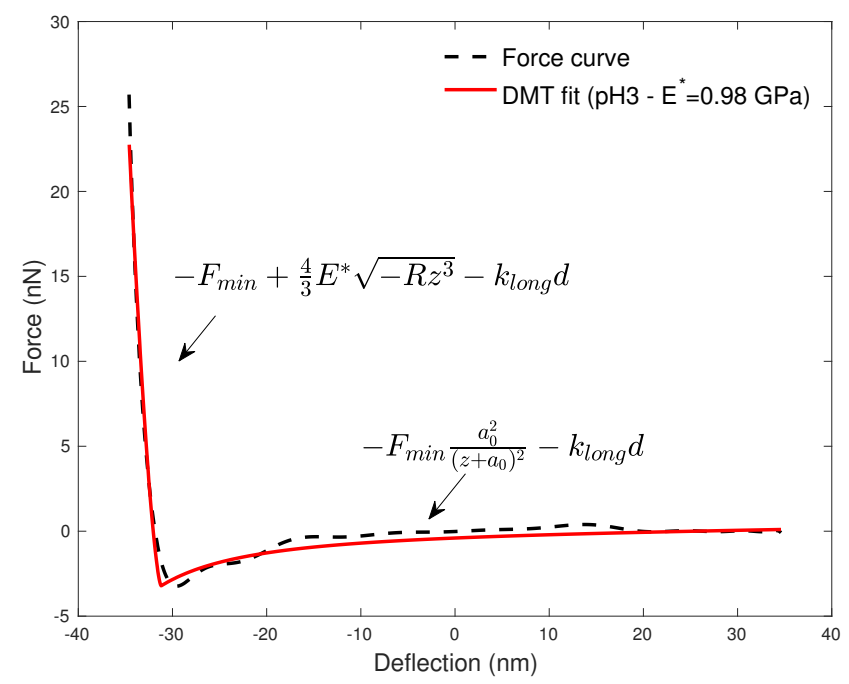

Figure 2: Force-deflection curve acquired on the surface of the pH3 ELP sample. The red curve is the result of the fitting procedure using Eq.1. 


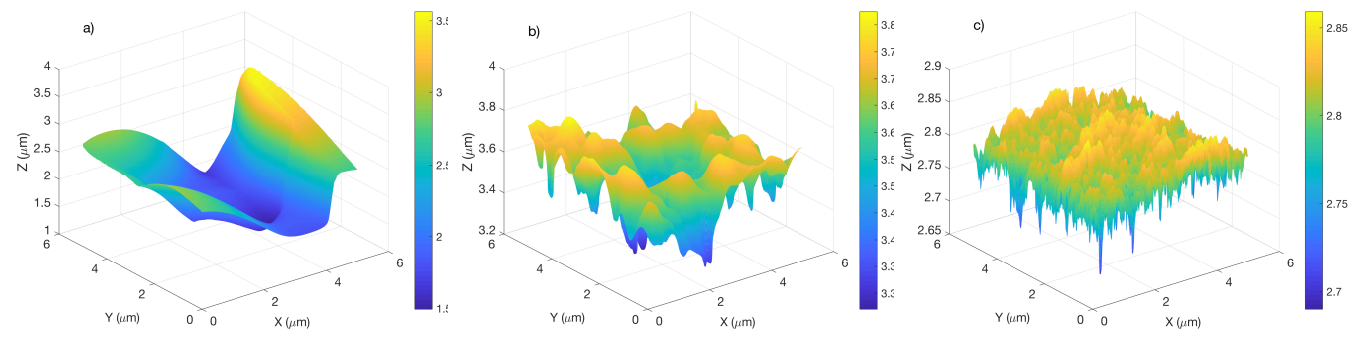

Figure 3: AFM images (scan area $5 \mu \mathrm{mx} 5 \mu \mathrm{m}$ ) of the surfaces of the ELP sample prepared at a) $\mathrm{pH} 3$, b) $\mathrm{pH} 6$ and c) $\mathrm{pH} 9$. 


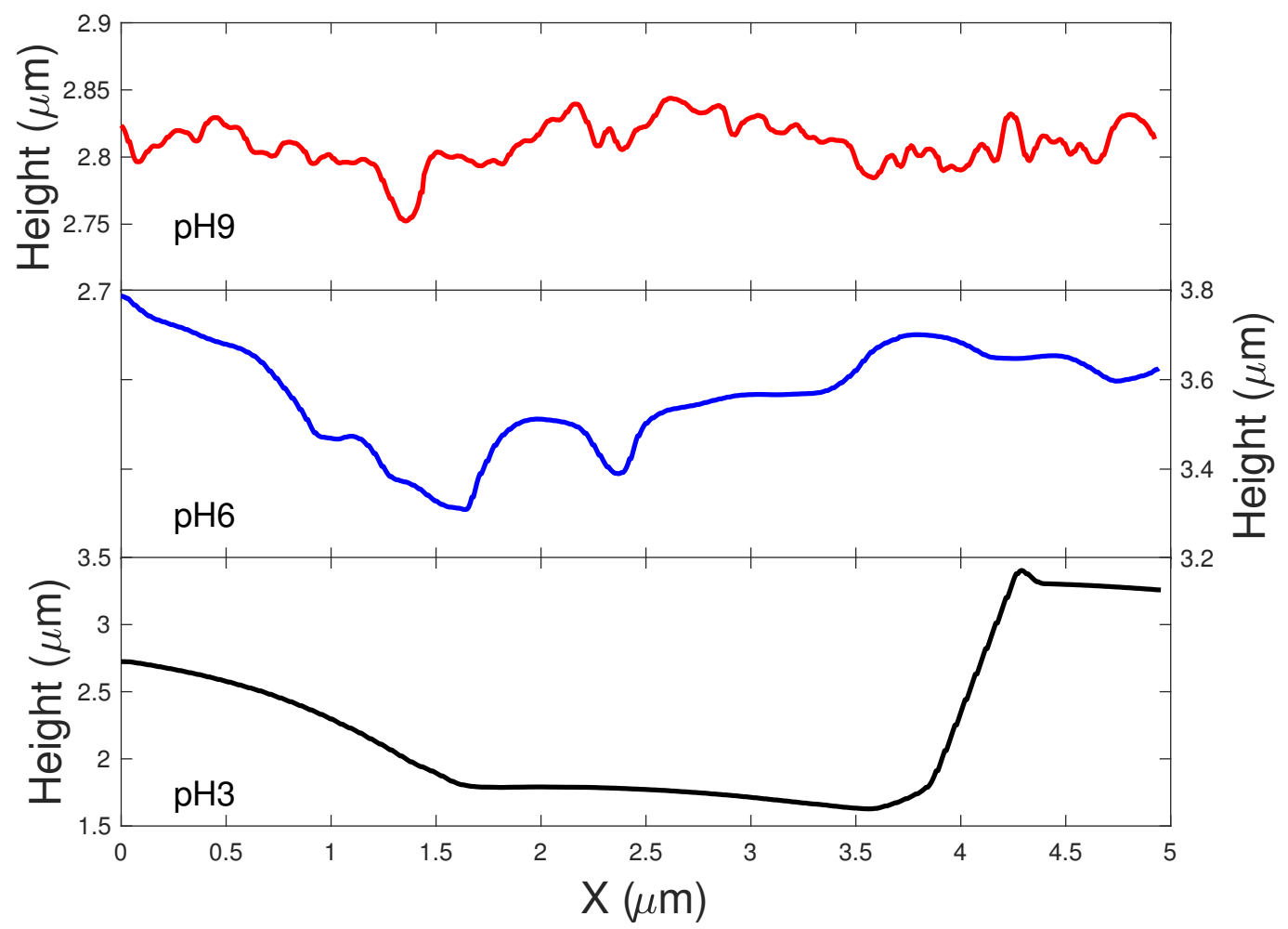

Figure 4: Line profile along the surface of the ELP samples produced at different $\mathrm{pH}$ values. 

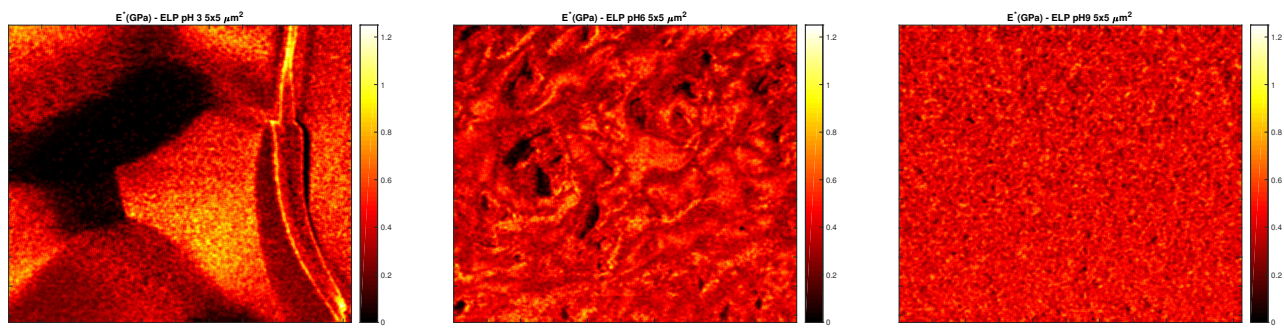

Figure 5: Maps of the elastic modulus $\mathrm{E}^{*}$ obtained fitting the force curves using the DMT model. Maps in panel a), b) and c) correspond to the scanned areas reported in Fig.3. 

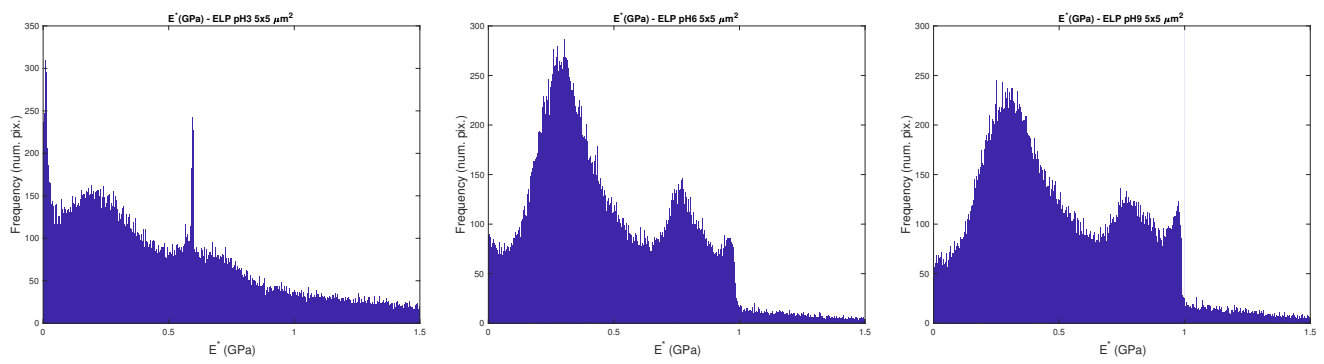

Figure 6: Histogram of distribution function of elastic modulus for ELP synthesized for different $\mathrm{pH} 3,6$ and 9 


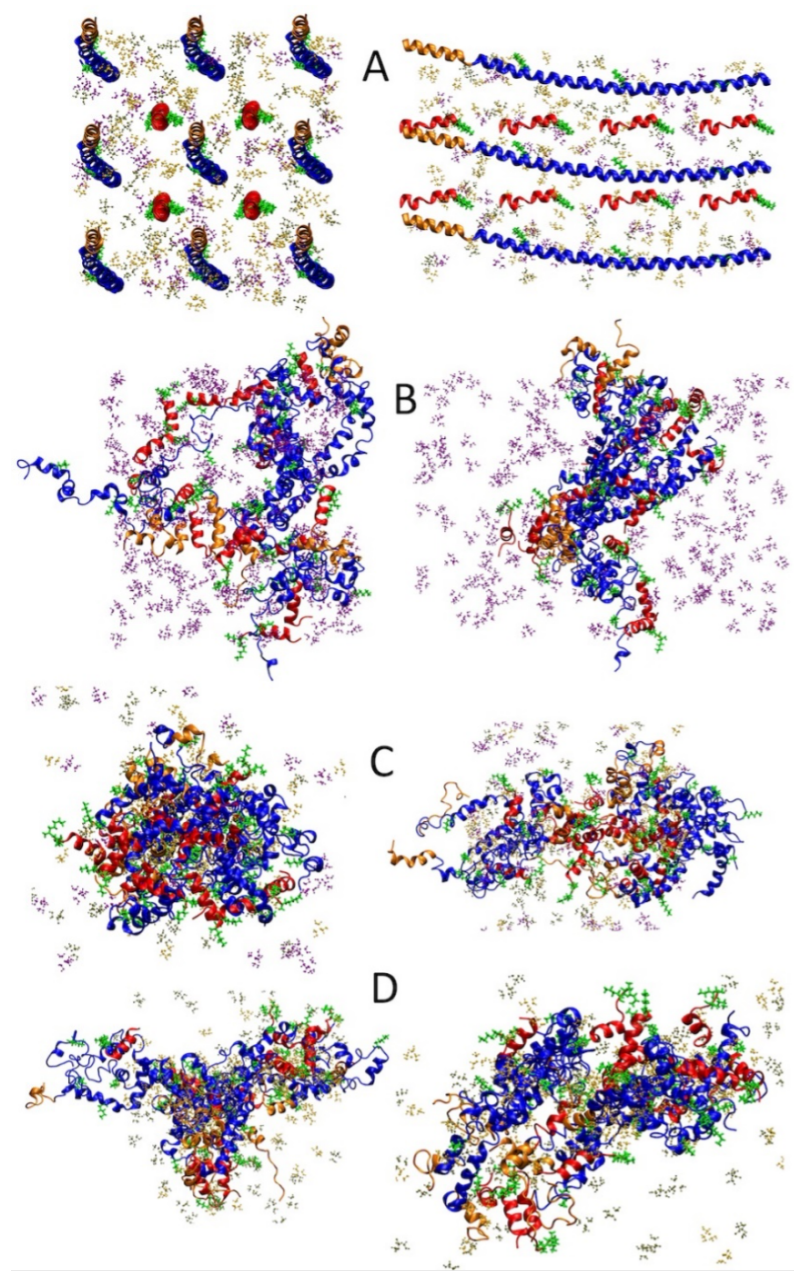

Figure 7: A) Top and B) side view of the initial configuration of the system for $\mathrm{pH}$ 7. The protein content and position are the same for all three $\mathrm{pH}$ value systems. The cell binding and structural binding sites are covalently connected. The initial configuration of proteins is set to $\alpha$-helical configuration. Cell binding peptides are in orange, structural binding peptides are in blue and QK peptides are in red. LYS amino acids are in green. THPC is in purple, TrHP is in orange and TrHPO is in tan. 

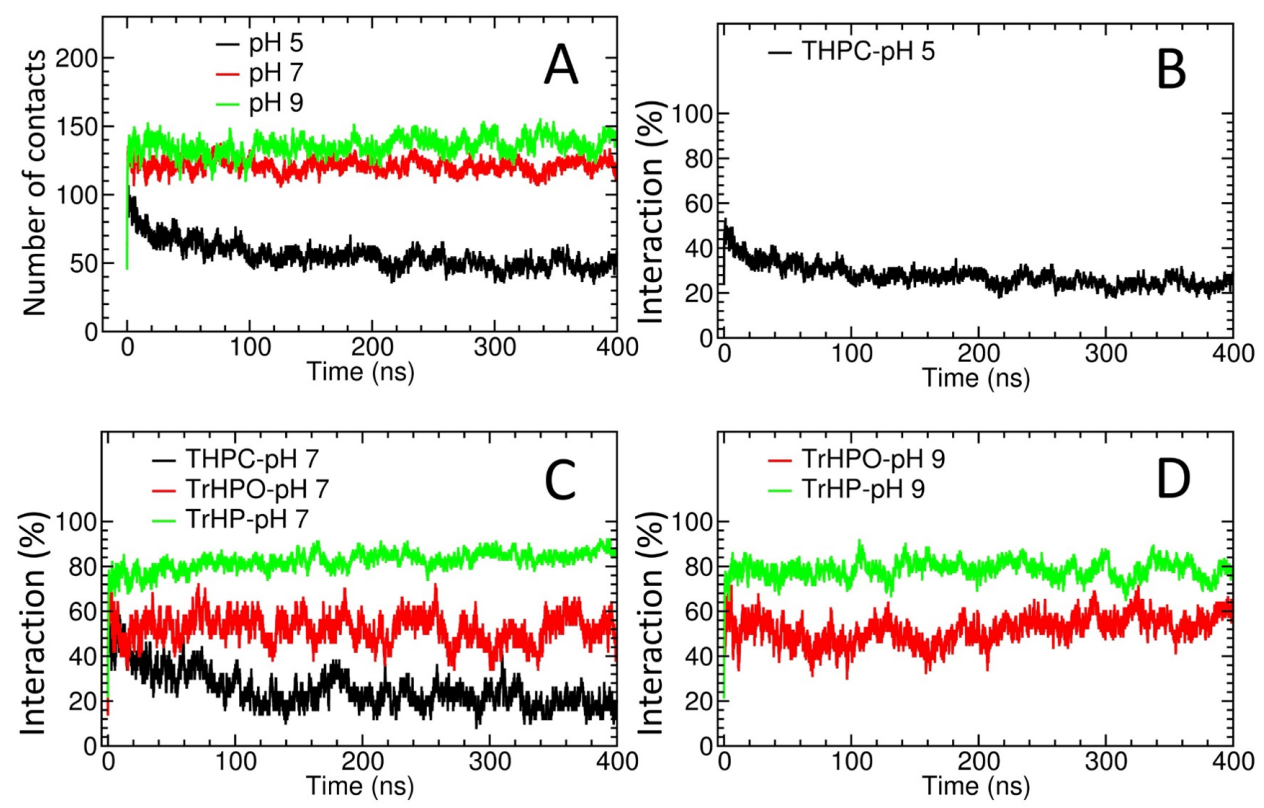

Figure 8: Interactions between the cross-linkers and proteins. A) Number of total contacts at different $\mathrm{pH}$ values, $\mathrm{B}$ ) percent of THPC crosslinkers interacting with proteins at $\mathrm{pH} 5$, C) percent of different crosslinkers interacting with proteins at $\mathrm{pH} 7$ and $\mathrm{D}$ ) percent of different crosslinkers interactingwith proteins at $\mathrm{pH} 9$. 


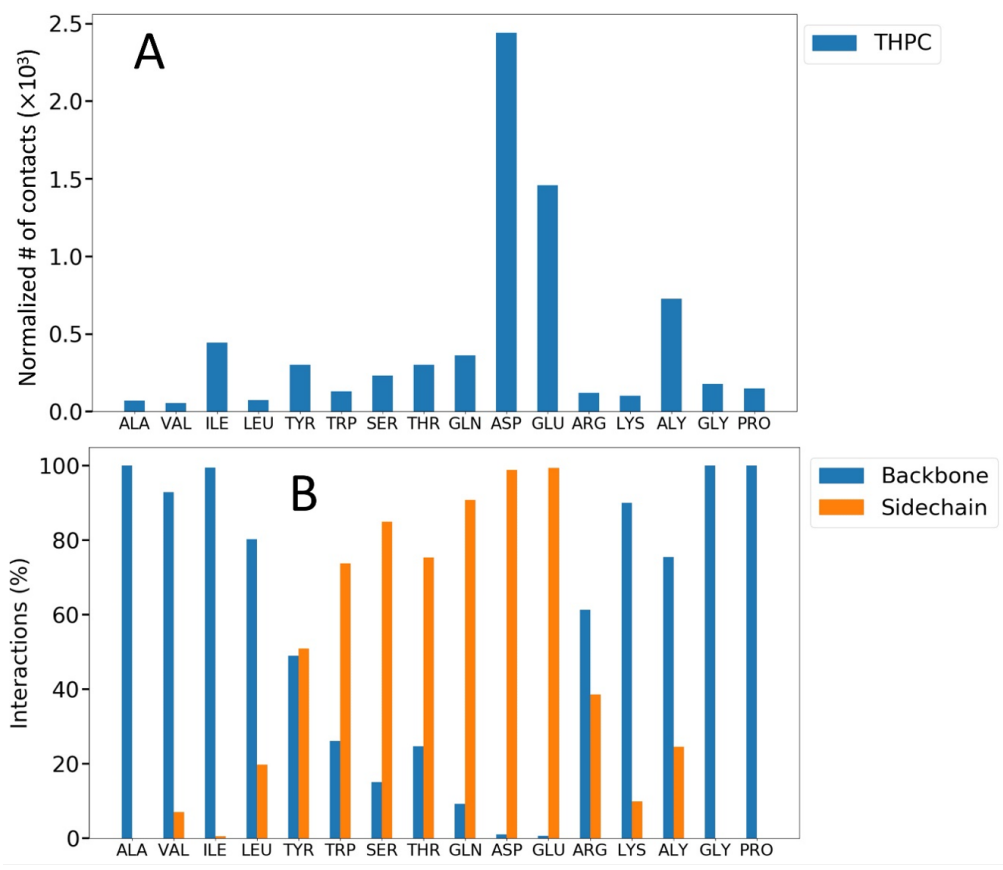

Figure 9: Cross-linkers at $\mathrm{pH} 5$ interacting with different amino acids of the protein mixture. A) Number of contacts between THPC cross-linkers and different amino acids residues in the system and $\mathrm{B}$ ) ratio of THPC interacting with the side chain vs backbone of each amino acid. The values in (A) are normalized by the number of amino acids in the system and the number of cross-linkers in the system. 

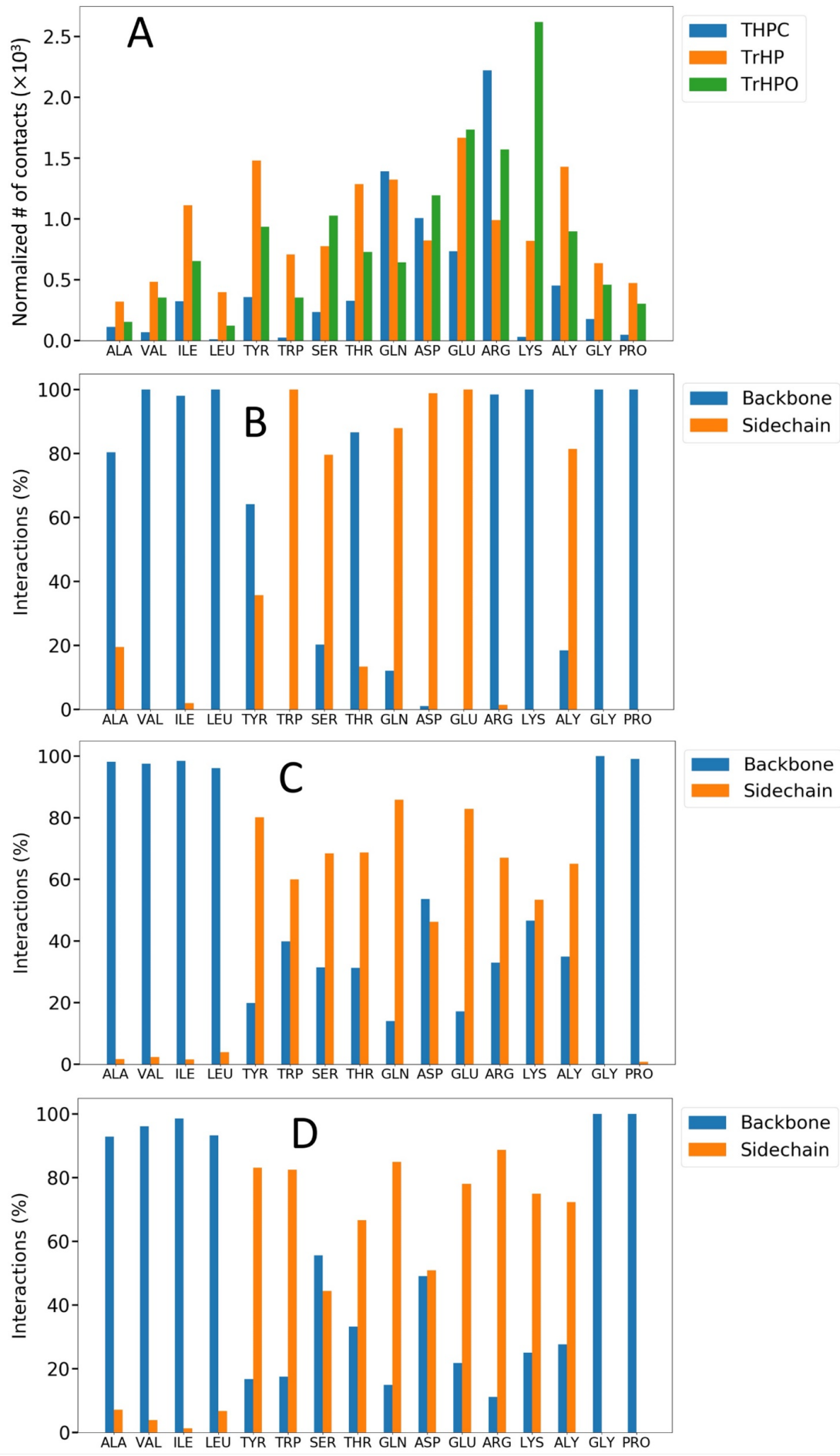

Figure 10: Cross-linkers at $\mathrm{pH} 7$ interactigg with different amino acids of the protein mixture. A) Number of contacts between THPC, TrHP and TrHPO cross-linkers and different amino acids residues in the system, B) ratio of THPC interacting with the side chain vs backbone of each amino acid, C) ratio of TrHP interacting with the side chain vs backbone of each amino acid and D) ratio of TrHPO interacting with the side chain vs backbone of each amino acid. The values in (A) are normalized by the number of amino acids in the system and the number of cross-linkers in the system. 


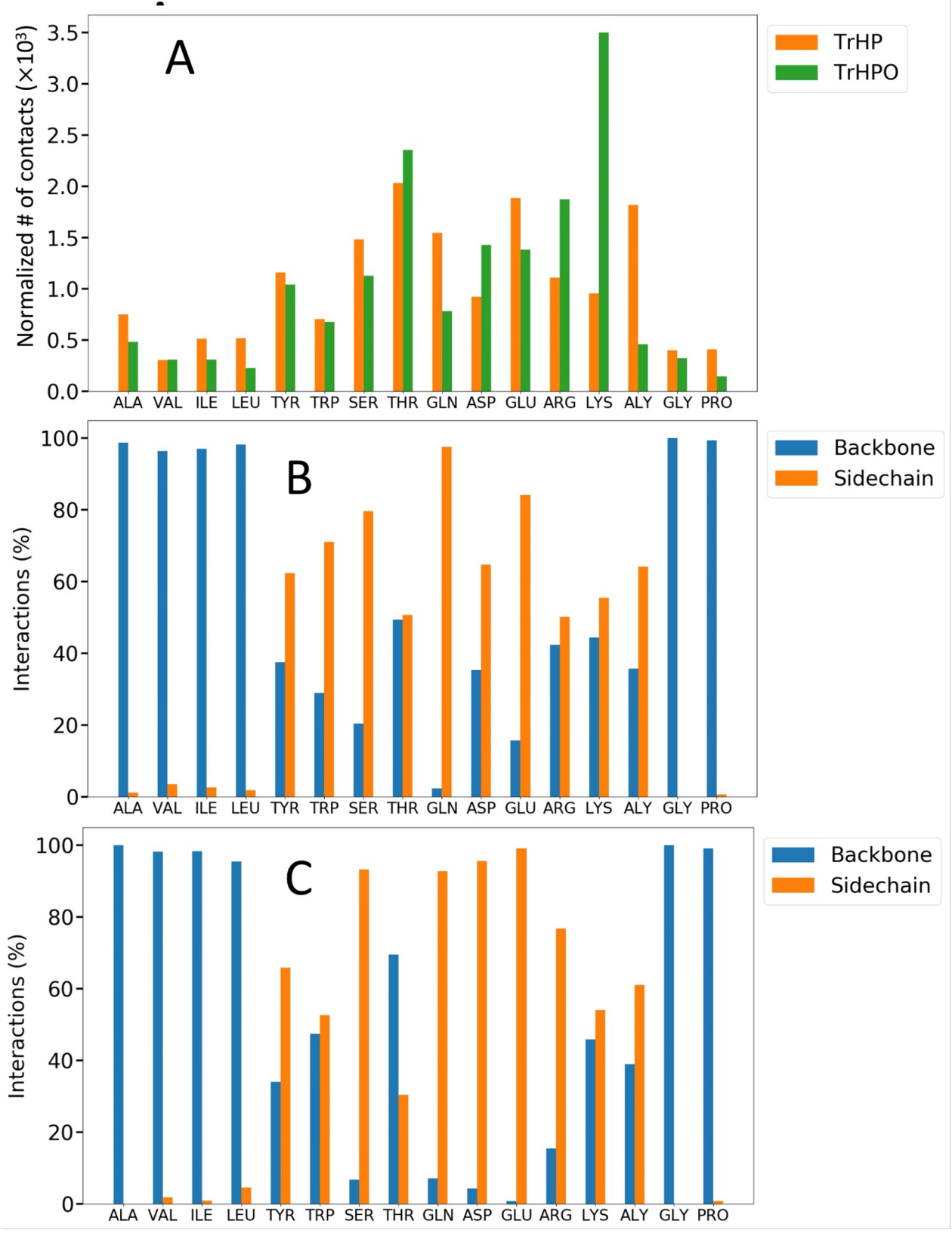

Figure 11: Cross-linkers at $\mathrm{pH} 9$ interacting with different amino acids of the protein mixture. A) Number of contacts between TrHP and TrHPO cross-linkers and different amino acids residues in the system, B) ratio of TrHP interacting with the side chain vs backbone of each amino acid and $\mathrm{C}$ ) ratio of TrHPO interacting with the side chain vs backbone of each amino acid. The values in (A) are normalized by the number of each amino acids in the system and the number of cross-linkers in the system. 

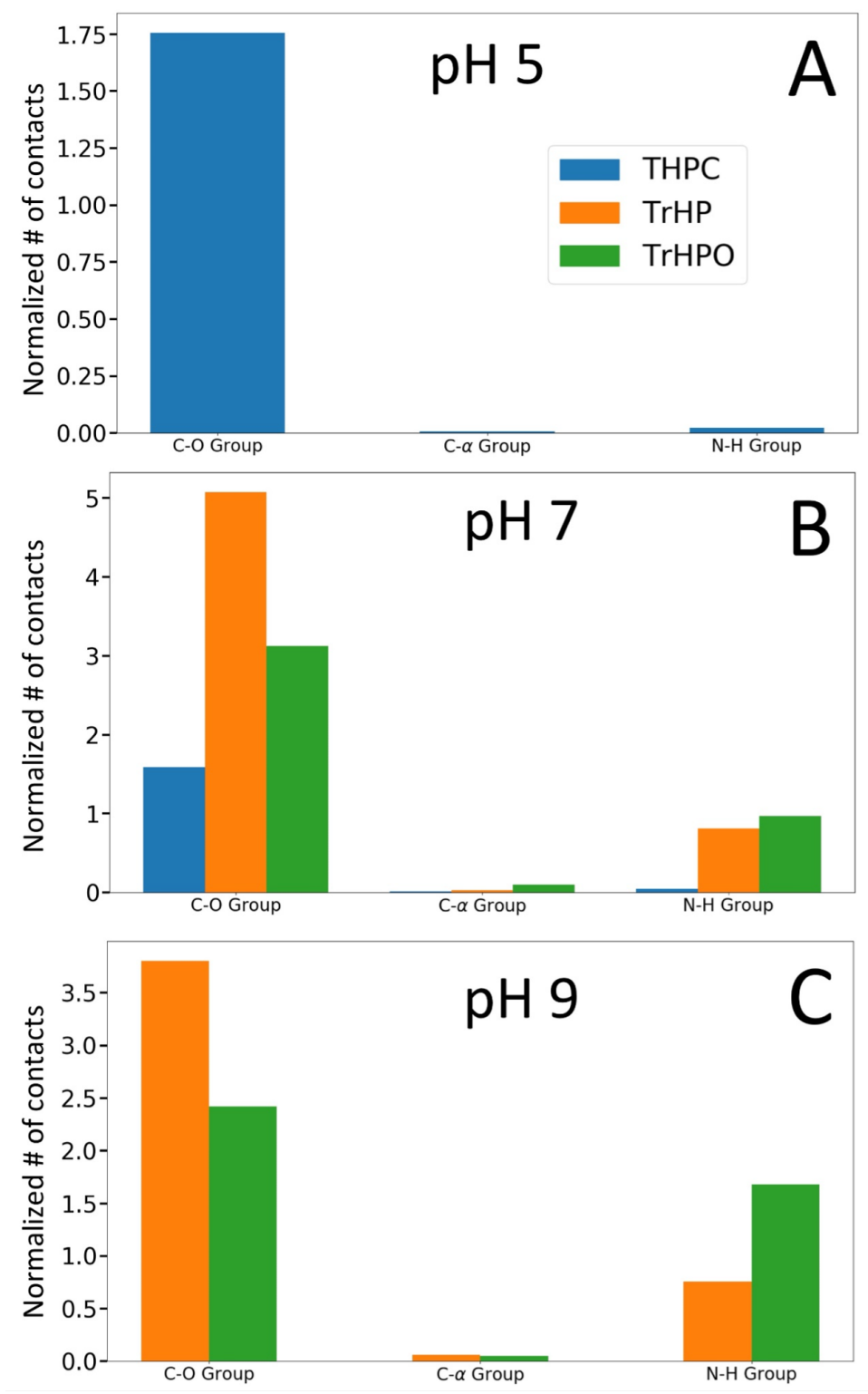

Figure 12: Number of contacts between different cross-linkers with different parts of protein backbone in different $\mathrm{pH}$ values. A) $\mathrm{pH} 5, \mathrm{~B}) \mathrm{pH} 7$ and $\mathrm{C}$ ) $\mathrm{pH} 9$. Number of contacts are normalized by total number of the cross-linker. 

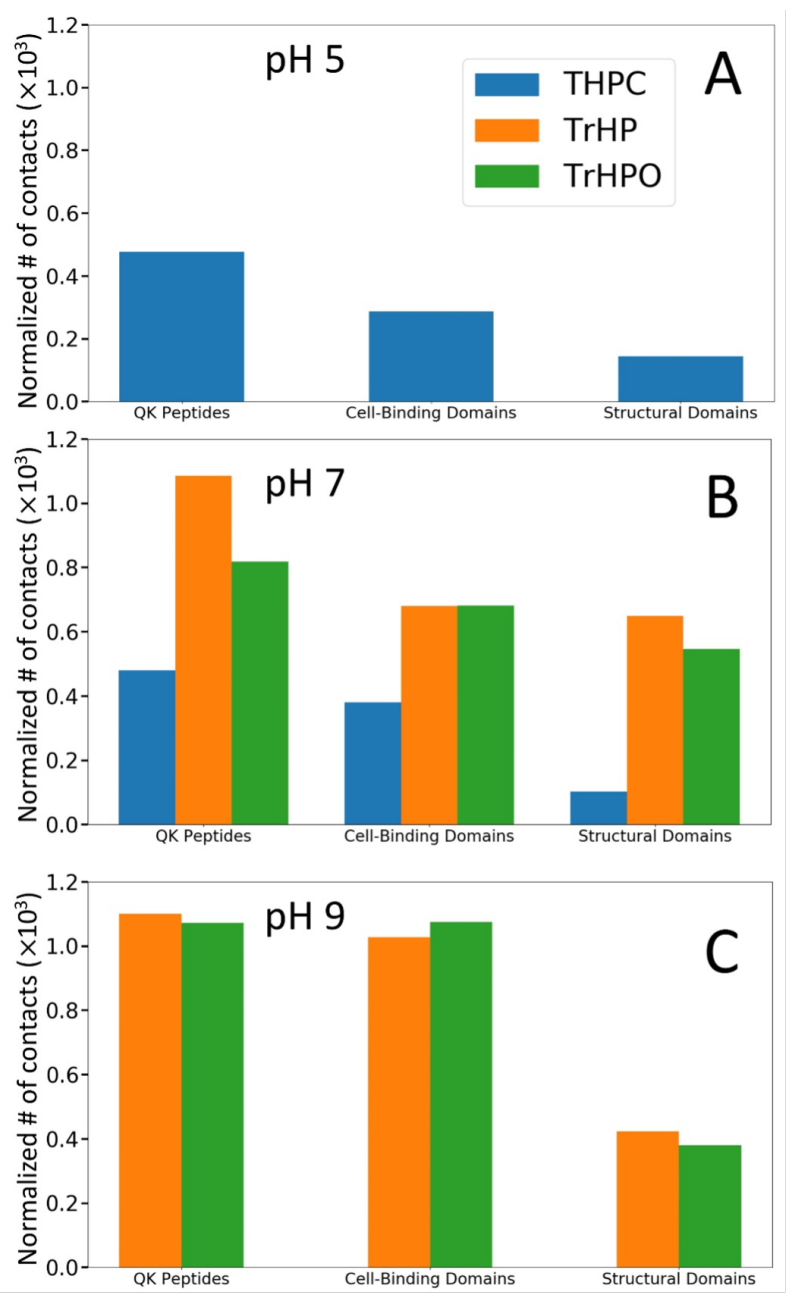

Figure 13: Number of contacts between different cross-linkers and different proteins in the system at different $\mathrm{pH}$ values. A) $\mathrm{pH} 5, \mathrm{~B}) \mathrm{pH} 7$ and C) pH9. Each cross-linker interaction is normalized by total number of the cross-linker. For each peptide, the number of interactions is normalized by number of the same type peptides, as well as the number of amino acids on each peptide. 


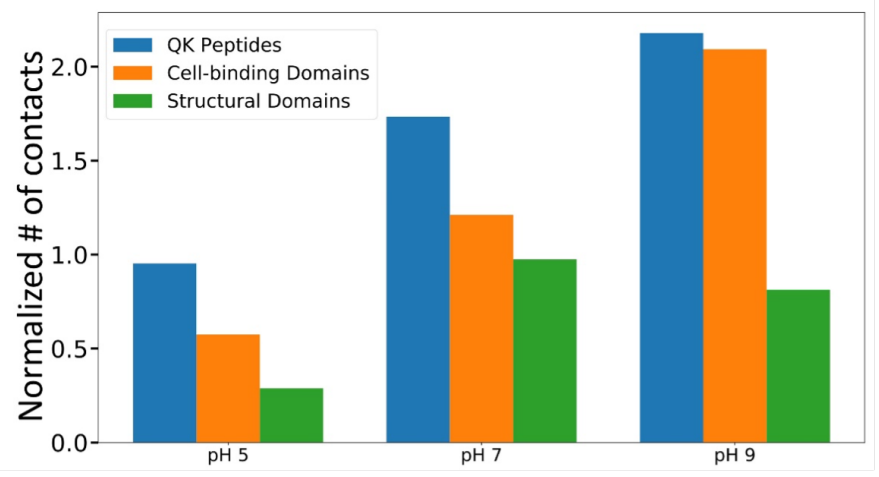

Figure 14: Number of contacts between all cross-linkers and different proteins in the system at different $\mathrm{pH}$ values. The values are normalized by total number of the cross-linkers and total the number of amino acids on each peptide. 

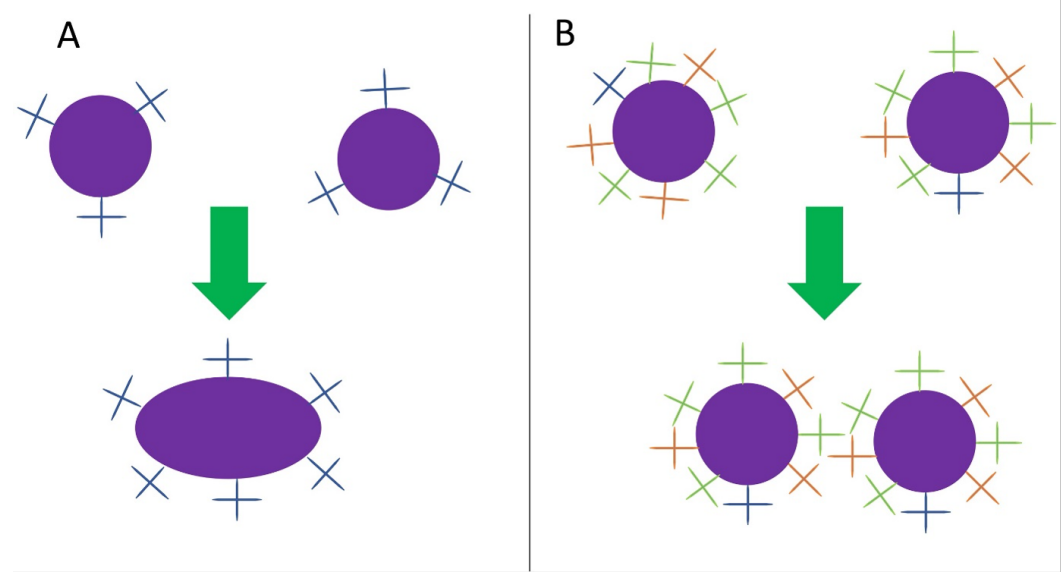

Figure 15: Schematics on the role of cross-linkers and proteins in producing the ELP configurations in different $\mathrm{pH}$ values. At low $\mathrm{pH}$ values (A), lower number of cross-linkers have covered the protein, while at higher $\mathrm{pH}$ values, (B) a greater number of cross-linkers have covered the protein. The aggregated proteins are shown as purple circles. The cross-linkers are shown as cross sign in different colors.. 